\title{
Thermohydrodynamics in Quantum Hall Systems
}

\author{
Hiroshi AKerA* and Hidekatsu SuzuurA ${ }^{\dagger}$ \\ Department of Applied Physics, Graduate School of Engineering, Hokkaido University, Sapporo 060-8628
}

(Received July 7, 2018)

A theory of thermohydrodynamics in two-dimensional electron systems in quantizing magnetic fields is developed including a nonlinear transport regime. Spatio-temporal variations of the electron temperature and the chemical potential in the local equilibrium are described by the equations of conservation with the number and thermal-energy flux densities. A model of these flux densities due to hopping and drift processes is introduced for a random potential varying slowly compared to both the magnetic length and the phase coherence length. The flux measured in the standard transport experiment is derived and is used to define a transport component of the flux density. The equations of conservation can be written in terms of the transport component only. As an illustration, the theory is applied to the Ettingshausen effect, in which a one-dimensional spatial variation of the electron temperature is produced perpendicular to the current.

KEYWORDS: integer quantum Hall effect, Ettingshausen effect, nonlinear transport, local equilibrium, thermohydrodynamics, theory

\section{Introduction}

Studies on spatial and temporal variations of thermodynamic and hydrodynamic variables reveal important properties of the studied system. ${ }^{1,2}$ A length scale and a time scale of variations, which occur in response to an external perturbation as well as in self-organization, are fundamental quantities which are never obtained by studying the system in uniform steady states.

Studies in this direction have already been performed by many authors in two-dimensional electron systems in quantizing magnetic fields, which exhibit the quantum Hall effect in the region of low temperatures and small currents. ${ }^{3,4}$ Theoretical $^{5}$ and experimental studies on spatial variations of the electrostatic potential in equilibrium have revealed a unique spatial pattern consisting of compressible and incompressible strips, which is a manifestation of the Landau quantization. These studies have recently been extended to nonequilibrium states with the applied current, and the response of the electrostatic potential to the applied current has been investigated experimentally ${ }^{6}$ and theoretically. ${ }^{7}$ However, most of these previous works have been restricted to quantities related to the chemical potential and the electrostatic potential. The electron temperature is known to be the key variable in the large-current regime where the breakdown of the quantum Hall effect ${ }^{8-10}$ takes place. The spin density is the order parameter of the ferromagnetic phase which appears when the Landau level filling factor is an odd integer. For a complete description of thermohydrodynamics in quantum Hall systems, studies need to be extended to spatial variations of such variables and to their temporal variations.

Spatio-temporal variations of the electron temperature have been investigated indirectly by measuring spatial $^{11-13}$ and temporal ${ }^{9,14,15}$ variations of the diagonal component of the resistivity tensor. Kawano and

*E-mail address: akera@eng.hokudai.ac.jp

${ }^{\dagger}$ E-mail address: suzuura@eng.hokudai.ac.jp
Komiyama ${ }^{16,17}$ have recently investigated the electron temperature more directly by measuring the local cylotron emission intensity using a scanning optical probe, and have revealed a variety of spatial variations of the electron temperature. The first attempt to construct a general theory for spatio-temporal variations of the electron temperature and the chemical potential in quantum Hall systems was made by one of the present authors. ${ }^{18}$ However, this previous theory has been found to employ an inexact formula of the thermal flux density.

In this paper we develop a theory of thermohydrodynamics in quantum Hall systems, which is a revision and a generalization of the previous theory. ${ }^{18}$ We describe spatio-temporal variations of the electron temperature and the chemical potential in the local equilibrium including the nonlinear transport regime with use of the equations of conservation, as in the previous theory. ${ }^{18} \mathrm{~A}$ revision is made by writing the equations in terms of the total thermal-energy flux density due to the drift motion. We generalize the previous theory to study thermohydrodynamics in the presence of a nonuniform potential such as a slowly-varying confining potential.

The organization of the paper is as follows. In $\S 2$, we introduce a model, assumptions, and macroscopic variables. Random potential due to ionized impurities is assumed to be slowly varying in the scale of both the magnetic length and the phase coherence length. In $\S 3$, we describe the equations of conservation and introduce a model of the number and thermal-energy flux densities due to hopping and drift processes. Each of the flux densities is a linear function of gradients of the electron temperature, the electrochemical potential, and the electrostatic potential. The coefficients in these linear functions depend on the local electron temperature and the local chemical potential. Such dependences are the origin of nonlinearity in this theory. In $\S 4$, we derive formulas for fluxes measured in the standard transport experiment for a narrow sample with a steep confining potential. In $\S 5$, 
we define a transport flux density for each of the measured fluxes. We show that the transport flux density defined in this manner is equal to the total flux density minus the magnetization current density. We find that the equations of conservation can be written in terms of the transport component only. It is shown that the transport flux densities satisfy the Einstein relation and the Onsager relation, while the total flux densities do not. In $\S 6$, as an illustration, we apply our theory to the Ettingshausen effect, in which a one-dimensional spatial variation of the electron temperature is produced perpendicular to the current. In $\S 7$, we review briefly the existing literature on the thermoelectric-transport theory in quantizing magnetic fields and on thermohydrodynamics in quantum Hall systems. In $\S 8$, conclusions are given.

\section{Model, Assumptions and Macroscopic Vari- ables}

\subsection{Drift and Hopping Processes}

We consider a two-dimensional electron system (in the $x y$ plane) in a perpendicular magnetic field $\boldsymbol{B}=(0,0, B)$. The local potential energy $V_{\text {loc }}$ consists of the disorder potential due to ionized donors with the fluctuation length scale of $l_{\mathrm{vh}} \sim 0.1 \mu \mathrm{m}^{19}$ and the macroscopic potential $V$. The macroscopic potential $V$ consists of the potential in equilibrium such as the confining potential and that induced by the applied current. The fluctuation length scale $l_{\mathrm{vh}}$ is much longer than the magnetic length $l=\sqrt{\hbar c / e|B|}(e>0)$ which is about $0.01 \mu \mathrm{m}$ at $|B|=5 \mathrm{~T}$.

An electron loses the phase coherence by a scattering from other electrons and phonons. The phase coherence length is assumed to be less than $l_{\mathrm{vh}}$. Therefore the Anderson localization due to the interference in the disorder potential as well as the energy quantization for closed orbits around potential hills and valleys are negligible. Then we employ the classical-drift model, ${ }^{20-22}$ in which each electron state is described by a wave packet with extent of the order of $l$ drifting along the equipotential line. The energy of the wave packet at $\boldsymbol{r}_{\mathrm{wp}}$ in the Landau level specified by a set of quantum numbers $\alpha=\{N, \sigma\}$ with the Landau index $N=0,1,2, \cdots$ and spin $\sigma$ is given by

$$
\varepsilon_{\alpha}^{0}+V_{\mathrm{loc}}\left(\boldsymbol{r}_{\mathrm{wp}}\right)
$$

where $\varepsilon_{\alpha}^{0}$ is the energy of the Landau level in the absence of the potential. The wave packet follows the equipotential line of $V_{\text {loc }}$ with a velocity given by

$$
\frac{d \boldsymbol{r}_{\mathrm{wp}}}{d t}=\frac{l^{2}}{\hbar} s_{B} \hat{\epsilon} \boldsymbol{\nabla} V_{\mathrm{loc}}\left(\boldsymbol{r}_{\mathrm{wp}}\right), s_{B}=\frac{B}{|B|}, \hat{\epsilon}=\left(\begin{array}{cc}
0 & 1 \\
-1 & 0
\end{array}\right) \text {. }
$$

A wave packet with energy at the center of the broadened Landau level follows the extended equipotential line of the disorder potential and contributes to the macroscopic current by the drift motion given by eq.(2). Other wave packets follow closed orbits within each vh-region. Here a vh-region is a subspace which is bounded by extended equipotential lines and contains one potential hill or valley. A hopping of such a localized wave packet between neighboring vh-regions by a scattering from other electrons contributes to the macroscopic current. ${ }^{23} \mathrm{~A}$ tunneling through a saddle point of the potential also produces the macroscopic current, although its contribution is estimated to be small. ${ }^{24}$ Compared to such intra-Landau-level transitions, the contribution of interLandau-level transitions to the macroscopic current is negligible in the slowly-varying potential. ${ }^{23}$ In this paper we consider the drift and the hopping processes within each Landau level in calculating the macroscopic current.

\subsection{Local Equilibrium}

We employ the local-equilibrium approximation, which has been used extensively in various systems and is described in detail, for example, in refs.1 and 25. We assume the local equilibrium within each vh-region, which means that the energy distribution of electrons in each vh-region is described by the Fermi distribution function with the electron temperature $T_{\mathrm{e}}$ and the electrochemical potential $\mu_{\mathrm{ec}}$ of the vh-region:

$$
f\left(\varepsilon, \mu_{\mathrm{ec}}, T_{\mathrm{e}}\right)=1 /\left\{\exp \left[\left(\varepsilon-\mu_{\mathrm{ec}}\right) / k_{\mathrm{B}} T_{\mathrm{e}}\right]+1\right\} .
$$

Each of $T_{\mathrm{e}}$ and $\mu_{\mathrm{ec}}$ differs from vh-region to vh-region in nonequilibrium states. The same electron distribution within the condition of constant $T_{\mathrm{e}}$ has been used in a theory on the quantum Hall effect. ${ }^{20}$ To maintain the local equilibrium, it is necessary that the applied current and the magnetic field should vary slowly in time compared with the time necessary for the relaxation to the local equilibrium. The slowest process involved in this relaxation is the transition of electrons between Landau levels in the case where the distribution of electrons to different Landau levels deviates from that in the local equilibrium. We also assume that phonons are in equilibrium with the lattice temperature $T_{\mathrm{L}}$, and that $T_{\mathrm{L}}$ does not change by the presence of the applied current.

\subsection{Spatial Averaging and Macroscopic Variables}

Since the potential due to ionized donors is random, quantities such as electron and energy densities fluctuate in the length scale of $l_{\mathrm{vh}} \sim 0.1 \mu \mathrm{m}$. We eliminate such random fluctuations by a spatial averaging, while we retain variations in the scale larger than $l_{\mathrm{vh}}$ which are produced by the confining potential and by the applied current. We assume that $T_{\mathrm{e}}$ and $\mu_{\mathrm{ec}}$ in nonequilibrium vary slowly in space compared with $l_{\mathrm{vh}}$. We note that the spatial variation of the measured cyclotron emission intensity is also macroscopic because of the limited spatial resolution of $50 \mu \mathrm{m} .{ }^{17}$

Thermohydrodynamics studies spatio-temporal variations of macroscopic variables. The macroscopic variables in the present system are the electron temperature $T_{\mathrm{e}}(\boldsymbol{r}, t)$, the electrochemical potential $\mu_{\mathrm{ec}}(\boldsymbol{r}, t)$, and the potential energy $V(\boldsymbol{r}, t)$. The chemical potential $\mu(\boldsymbol{r}, t)$ is defined by $\mu=\mu_{\mathrm{ec}}-V$. The macroscopic electric field $\boldsymbol{E}(\boldsymbol{r}, t)$ is given by $\boldsymbol{E}=\boldsymbol{\nabla} V / e$. The macroscopic potential $V(\boldsymbol{r}, t)$ is determined by electrostatics in terms of the averaged electron density which is a function of $T_{\mathrm{e}}$ and $\mu$. Other macroscopic variables $T_{\mathrm{e}}$ and $\mu_{\mathrm{ec}}$ are determined by the following equations of conservation. 


\section{Formulation with the Total Fluxes}

\subsection{Equations with the Total Flux Densities}

There are two equations of conservation in our model of quantum Hall systems. One describes the conservation of the electron number, and the other describes the conservation of the energy. The equation of the momentum conservation is absent since in the classical-drift model the velocity of the wave packet is not an additional degree of freedom but given in eq.(2) to be a function of its position.

The equation of the electron number conservation is

$$
\frac{\partial n}{\partial t}=-\nabla \cdot \boldsymbol{j}_{n},
$$

where $n$ is the electron density and $\boldsymbol{j}_{n}$ is the number flux density. The equation of the energy conservation is

$$
\frac{\partial \epsilon}{\partial t}=-\nabla \cdot \boldsymbol{j}_{\epsilon}-P_{\mathrm{L}}
$$

Here $\epsilon$ is the energy density and $\boldsymbol{j}_{\epsilon}$ is the energy flux density, while $P_{\mathrm{L}}$ is the energy loss per unit area at point $\boldsymbol{r}$ due to the heat transfer between electrons and phonons and is in general a function of $\mu, T_{\mathrm{e}}$, and $T_{\mathrm{L}}$. The energy density is the sum of the kinetic energy density and the potential energy density: $\epsilon=\epsilon_{\text {kin }}+n V$.

Equations describing the time evolution of $\mu$ and $T_{\mathrm{e}}$ are derived from those of $n\left(\mu, T_{\mathrm{e}}\right)$ and the entropy density $s\left(\mu, T_{\mathrm{e}}\right)$, respectively. The equation for the time evolution of $s$ is derived using eqs. (4), (5), and

$$
T_{\mathrm{e}} d s=d \epsilon-\mu_{\mathrm{ec}} d n,
$$

to be

$$
T_{\mathrm{e}} \frac{\partial s}{\partial t}=-\nabla \cdot \boldsymbol{j}_{q}-\nabla \mu_{\mathrm{ec}} \cdot \boldsymbol{j}_{n}-P_{\mathrm{L}}
$$

where $\boldsymbol{j}_{q}$ is the thermal flux density defined by

$$
\boldsymbol{j}_{q}=\boldsymbol{j}_{\epsilon}-\mu_{\mathrm{ec}} \boldsymbol{j}_{n}
$$

\subsection{Number and Thermal Flux Densities}

\subsubsection{Hopping Components}

First we consider the number flux between neighboring vh-regions due to hopping processes, denoted as $J_{n}^{\text {hop }}$. Since we consider only hopping processes within each Landau level, we have $J_{n}^{\text {hop }}=\sum_{\alpha} J_{n \alpha}^{\text {hop }}$. Each $J_{n \alpha}^{\text {hop }}$ is induced by the difference in the electron temperature $\Delta T_{\mathrm{e}}$ and that in the electrochemical potential $\Delta \mu_{\mathrm{ec}}$ between the two vh-regions. In the first order of $\Delta T_{\mathrm{e}}$ and $\Delta \mu_{\mathrm{ec}}$, $J_{n \alpha}^{\text {hop }}$ is given by

$$
J_{n \alpha}^{\text {hop }}=A_{\alpha} \Delta \mu_{\mathrm{ec}}+B_{\alpha} \Delta T_{\mathrm{e}}
$$

In the following we show that the coefficients $A_{\alpha}$ and $B_{\alpha}$ are related to each other in our model of hopping processes.

The wave packets contributing to the hopping processes are only those in the vicinity of the boundary between the two vh-regions, since the transition rate is negligible when the distance between the wave packets is much larger than $l$. The energies of such wave packets are confined within an energy range around $\varepsilon_{\alpha}(\boldsymbol{r})$ with width $\Gamma_{\text {hop }}$ where $\varepsilon_{\alpha}(\boldsymbol{r})=\varepsilon_{\alpha}^{0}+V(\boldsymbol{r})$ and $\Gamma_{\text {hop }} \sim \Gamma l / l_{\text {vh }}$ with $\Gamma$ the width of the broadened Landau level. Therefore the corresponding occupation probability is well approximated to be

$$
f_{\alpha}=f\left(\varepsilon_{\alpha}, \mu_{\mathrm{ec}}, T_{\mathrm{e}}\right),
$$

when $\Gamma_{\text {hop }} \ll k_{\mathrm{B}} T_{\mathrm{e}}$. In the case of elastic hopping processes between a vh-region with $\mu_{\mathrm{ec}}$ and $T_{\mathrm{e}}$ and its neighbor with $\mu_{\mathrm{ec}}+\Delta \mu_{\mathrm{ec}}$ and $T_{\mathrm{e}}+\Delta T_{\mathrm{e}}, J_{n \alpha}^{\mathrm{hop}}$ is given by

$J_{n \alpha}^{\mathrm{hop}}=-C_{\alpha}\left[f\left(\varepsilon_{\alpha}, \mu_{\mathrm{ec}}+\Delta \mu_{\mathrm{ec}}, T_{\mathrm{e}}+\Delta T_{\mathrm{e}}\right)-f\left(\varepsilon_{\alpha}, \mu_{\mathrm{ec}}, T_{\mathrm{e}}\right)\right]$.

In the first order of $\Delta T_{\mathrm{e}}$ and $\Delta \mu_{\mathrm{ec}}$, we obtain

$$
J_{n \alpha}^{\text {hop }}=-C_{\alpha}\left(\frac{\partial f_{\alpha}}{\partial \mu_{\mathrm{ec}}} \Delta \mu_{\mathrm{ec}}+\frac{\partial f_{\alpha}}{\partial T_{\mathrm{e}}} \Delta T_{\mathrm{e}}\right) .
$$

Also in the case of inelastic electron-electron scatterings, this formula is approximately applicable as long as the energy change in each hopping process is much smaller than $k_{\mathrm{B}} T_{\mathrm{e}}$. We assume eq.(12) in the following.

The hopping number flux density $\boldsymbol{j}_{n}^{\text {hop }}$ averaged in the macroscopic scale is then given by

$$
\boldsymbol{j}_{n}^{\mathrm{hop}}=-\sum_{\alpha} D_{\alpha}\left(\frac{\partial f_{\alpha}}{\partial \mu_{\mathrm{ec}}} \nabla \mu_{\mathrm{ec}}+\frac{\partial f_{\alpha}}{\partial T_{\mathrm{e}}} \nabla T_{\mathrm{e}}\right) .
$$

Here $D_{\alpha}$ is written in terms of the transition rate of each hopping process, which depends on the disorder potential. The disorder potential in turn is a function of $\mu$ and $T_{\mathrm{e}}$ since it is screened by electrons.

We introduce transport coefficients $L_{x x}^{11}$ and $L_{x x}^{12}$ and write $\boldsymbol{j}_{n}^{\text {hop }}$ as

$$
\boldsymbol{j}_{n}^{\text {hop }}=-L_{x x}^{11} \nabla \mu_{\mathrm{ec}}-L_{x x}^{12} T_{\mathrm{e}}^{-1} \nabla T_{\mathrm{e}}
$$

From eq.(13) we have

$$
\begin{gathered}
L_{x x}^{11}=e^{-2} \sigma_{x x}=\left(k_{\mathrm{B}} T_{\mathrm{e}}\right)^{-1} \sum_{\alpha} D_{\alpha} f_{\alpha}\left(1-f_{\alpha}\right), \\
L_{x x}^{12}=\left(k_{\mathrm{B}} T_{\mathrm{e}}\right)^{-1} \sum_{\alpha} D_{\alpha} f_{\alpha}\left(1-f_{\alpha}\right)\left(\varepsilon_{\alpha}^{0}-\mu\right) .
\end{gathered}
$$

When we assume the $N$ dependence of $D_{\alpha}$ to be $D_{\alpha}=$ $(2 N+1) D_{0}$ with $D_{0}$ the coefficient for $N=0$, the above formula of $L_{x x}^{11}$ coincides with that for short-range scatterings in the self-consistent Born approximation ${ }^{26}$ in the case where $\Gamma \ll k_{\mathrm{B}} T_{\mathrm{e}}$. Note that the above formula is applicable as long as $\Gamma_{\text {hop }} \ll k_{\mathrm{B}} T_{\mathrm{e}}$. The coefficients $L_{x x}^{11}$ and $L_{x x}^{12}$ are functions of $T_{\mathrm{e}}(\boldsymbol{r}, t)$ and $\mu(\boldsymbol{r}, t)$. In the linear-response regime, $L_{x x}^{11}$ and $L_{x x}^{12}$ are to be evaluated in equilibrium and eq.(14) reduces to the so-called phenomenological equation. ${ }^{1}$ In our model, a nonlinear effect is taken into account in dependences of $L_{x x}^{11}$ and $L_{x x}^{12}$ on deviations of $T_{\mathrm{e}}(\boldsymbol{r}, t)$ and $\mu(\boldsymbol{r}, t)$ from their equilibrium values.

The thermal flux density [eq.(8)] is given similarly, in hopping processes, by

$$
\boldsymbol{j}_{q}^{\mathrm{hop}}=-\sum_{\alpha}\left(\varepsilon_{\alpha}-\mu_{\mathrm{ec}}\right) D_{\alpha}\left(\frac{\partial f_{\alpha}}{\partial \mu_{\mathrm{ec}}} \nabla \mu_{\mathrm{ec}}+\frac{\partial f_{\alpha}}{\partial T_{\mathrm{e}}} \nabla T_{\mathrm{e}}\right) \text {. }
$$

This expresses that an electron in the Landau level $\alpha$ carries a thermal energy $\varepsilon_{\alpha}-\mu_{\mathrm{ec}}$. In terms of transport coefficients, $\boldsymbol{j}_{q}^{\text {hop }}$ is expressed as

$$
\boldsymbol{j}_{q}^{\text {hop }}=-L_{x x}^{21} \nabla \mu_{\mathrm{ec}}-L_{x x}^{22} T_{\mathrm{e}}^{-1} \nabla T_{\mathrm{e}}
$$


where

$$
\begin{gathered}
L_{x x}^{21}=L_{x x}^{12}, \\
L_{x x}^{22}=\left(k_{\mathrm{B}} T_{\mathrm{e}}\right)^{-1} \sum_{\alpha} D_{\alpha} f_{\alpha}\left(1-f_{\alpha}\right)\left(\varepsilon_{\alpha}^{0}-\mu\right)^{2} .
\end{gathered}
$$

\subsubsection{Drift Components}

The local flux density due to the drift motion fluctuates spatially since the local potential $V_{\text {loc }}$ contains the random potential. We average the local flux density to obtain the macroscopic flux density. The macroscopic number flux density in the Landau level $\alpha, \boldsymbol{j}_{n \alpha}^{\text {drift }}$, is written as

$$
\boldsymbol{j}_{n \alpha}^{\text {drift }}=\left\langle f\left(\varepsilon_{\alpha}^{0}+V_{\mathrm{loc}}, \mu_{\mathrm{ec}}, T_{\mathrm{e}}\right) h^{-1} s_{B} \hat{\epsilon} \boldsymbol{\nabla} V_{\mathrm{loc}}\right\rangle_{\mathrm{av}} .
$$

Since localized states make no contributions to the macroscopic flux density, the occupation probability of localized states in the above equation can be replaced by that of extended states. Then we have

$$
\boldsymbol{j}_{n \alpha}^{\mathrm{drift}}=f\left(\varepsilon_{\alpha}^{0}+V, \mu_{\mathrm{ec}}, T_{\mathrm{e}}\right)\left\langle h^{-1} s_{B} \hat{\epsilon} \boldsymbol{\nabla} V_{\mathrm{loc}}\right\rangle_{\mathrm{av}} .
$$

Since the spatial average of $\nabla V_{\text {loc }}$ is equal to $\nabla V$, then the number flux density $\boldsymbol{j}_{n}^{\text {drift }}$ due to all the Landau levels becomes

$$
\boldsymbol{j}_{n}^{\mathrm{drift}}=L_{y x}^{11} \hat{\epsilon} \nabla V
$$

with

$$
L_{y x}^{11}=\frac{\sigma_{y x}}{e^{2}}=\frac{s_{B}}{h} \sum_{\alpha} f_{\alpha} .
$$

Similarly we have for the thermal flux density

$$
\boldsymbol{j}_{q}^{\mathrm{drift}}=K_{y x}^{21} \hat{\epsilon} \nabla V
$$

with

$$
K_{y x}^{21}=\frac{s_{B}}{h} \sum_{\alpha}\left(\varepsilon_{\alpha}^{0}-\mu\right) f_{\alpha} .
$$

The coefficients $L_{y x}^{11}$ and $K_{y x}^{21}$ can be written as

$$
\begin{gathered}
L_{y x}^{11}=s_{B} \frac{2 \pi l^{2}}{h} n_{0}, \\
K_{y x}^{21}=s_{B} \frac{2 \pi l^{2}}{h}\left(\Omega_{0}+T_{\mathrm{e}} s_{0}\right),
\end{gathered}
$$

in terms of thermodynamic quantities $n_{0}, \Omega_{0}$, and $s_{0}$. Here $\Omega_{0}$ is the thermodynamic potential density corresponding to the grand canonical ensemble in the absence of disorder $\left(V_{\mathrm{loc}}=V\right)$ :

$\Omega_{0}\left(T_{\mathrm{e}}, \mu, B\right)=-\frac{k_{\mathrm{B}} T_{\mathrm{e}}}{2 \pi l^{2}} \sum_{\alpha} \ln \left[1+\exp \left(-\frac{\varepsilon_{\alpha}^{0}-\mu}{k_{\mathrm{B}} T_{\mathrm{e}}}\right)\right]$,

while $n_{0}$ and $s_{0}$ are the electron density and the entropy density, respectively, in this case:

$$
n_{0}=-\left(\frac{\partial \Omega_{0}}{\partial \mu}\right)_{T_{\mathrm{e}}, B}, \quad s_{0}=-\left(\frac{\partial \Omega_{0}}{\partial T_{\mathrm{e}}}\right)_{\mu, B} .
$$

\subsubsection{Total Flux Densities}

The total flux densities $\boldsymbol{j}_{n}=\boldsymbol{j}_{n}^{\text {hop }}+\boldsymbol{j}_{n}^{\text {drift }}$ and $\boldsymbol{j}_{q}=$ $\boldsymbol{j}_{q}^{\text {hop }}+\boldsymbol{j}_{q}^{\text {drift }}$ are written as

$$
\begin{aligned}
& j_{n x}=-L_{x x}^{11} \nabla_{x} \mu_{\mathrm{ec}}+L_{y x}^{11} \nabla_{y} V-L_{x x}^{12} T_{\mathrm{e}}^{-1} \nabla_{x} T_{\mathrm{e}}, \\
& j_{n y}=-L_{y x}^{11} \nabla_{x} V-L_{x x}^{11} \nabla_{y} \mu_{\mathrm{ec}}-L_{x x}^{12} T_{\mathrm{e}}^{-1} \nabla_{y} T_{\mathrm{e}}, \\
& j_{q x}=-L_{x x}^{12} \nabla_{x} \mu_{\mathrm{ec}}+K_{y x}^{21} \nabla_{y} V-L_{x x}^{22} T_{\mathrm{e}}^{-1} \nabla_{x} T_{\mathrm{e}}, \\
& j_{q y}=-K_{y x}^{21} \nabla_{x} V-L_{x x}^{12} \nabla_{y} \mu_{\mathrm{ec}}-L_{x x}^{22} T_{\mathrm{e}}^{-1} \nabla_{y} T_{\mathrm{e}} .
\end{aligned}
$$

The total flux densities are produced not only by $\nabla \mu_{\mathrm{ec}}$ and $\nabla T_{\mathrm{e}}$, but also by $\boldsymbol{\nabla} V$, and therefore they are in general nonzero in equilibrium. The standard transport experiment measures a flux through a cross section of the sample, which is zero in equilibrium. Such a flux, which is induced in nonequilibrium, is calculated in the next section.

\section{Measured Fluxes in a Narrow Wire}

\subsection{Model and Assumptions}

In this section we derive formulas for the fluxes measured in the standard transport experiment. We consider a narrow two-dimensional system along the $x$ direction and separate the system into the bulk region $0<y<\Delta y$ and the edge region $y<0, \Delta y<y$. In the edge region we assume the presence of a confining potential which increases to infinity so that the electron density decreases to zero within a length scale of the order of $l_{\mathrm{vh}}$. The width of the edge region $\Delta \eta$ is quite small and variations of $T_{\mathrm{e}}(x, y)$ and $\mu_{\mathrm{ec}}(x, y)$ within $\Delta \eta$ are negligible. Therefore we calculate the fluxes in the zeroth-order of $\Delta \eta$ (zero edge-width model). We also assume that the width of the bulk region $\Delta y$ is small compared with the length scale of variations of $T_{\mathrm{e}}$ and $\mu_{\mathrm{ec}}$, and calculate the fluxes in the first-order of $\Delta y$ (narrow wire model).

\subsection{Edge Currents}

We introduce coordinates $(\xi, \eta)$ for each boundary of the two-dimensional system. The unit vector normal to the boundary, directed to the outside of the sample, is denoted by $\boldsymbol{n}$. We take the $\eta$ axis in the direction of $\boldsymbol{n}$ and the $\xi$ axis along the boundary in the direction of $\hat{\epsilon} \boldsymbol{n}$.

We calculate fluxes in the edge region $\eta_{\text {edge }}<\eta<$ $\eta_{\text {edge }}+\Delta \eta$. In the region $\eta>\eta_{\text {edge }}+\Delta \eta$ the electron density and the flux densities are assumed to be negligible. Since $\Delta \eta$ is small in the present steep confining potential, the hopping flux in the edge region is negligible. The drift flux is not negligible because of the large gradient of the confining potential. With use of eq.(23), the drift number flux is given by

$$
\boldsymbol{J}_{n}^{\text {edge }}=K_{n} \hat{\epsilon} \boldsymbol{n},
$$

with

$$
K_{n}=\frac{s_{B}}{h} \sum_{\alpha} \int_{\eta_{\text {edge }}}^{\eta_{\text {edge }}+\Delta \eta} d \eta \frac{\partial V}{\partial \eta} f\left(\varepsilon_{\alpha}^{0}+V, \mu_{\mathrm{ec}}, T_{\mathrm{e}}\right) .
$$

Since the $\eta$ dependence of $\mu_{\mathrm{ec}}$ and $T_{\mathrm{e}}$ is neglected,

$$
K_{n}=\frac{s_{B}}{h} \sum_{\alpha} \int_{\varepsilon_{\alpha}}^{\infty} f\left(\varepsilon, \mu_{\mathrm{ec}}, T_{\mathrm{e}}\right) d \varepsilon=-s_{B} \frac{2 \pi l^{2}}{h} \Omega_{0},
$$


where $\varepsilon_{\alpha}, \mu_{\mathrm{ec}}, T_{\mathrm{e}}$ and $\Omega_{0}$ are to be evaluated at $\eta_{\text {edge }}$. The quantitiy $K_{n}$ is related to the magnetization per unit area, $M$, at $\eta_{\text {edge }}$ by

$$
K_{n}\left(\eta_{\text {edge }}\right)=(c / e) M\left(\eta_{\text {edge }}\right),
$$

since the edge electric current $\boldsymbol{I}$ and $M$ are related ${ }^{27}$ by $\boldsymbol{I}=-c M \hat{\epsilon} \boldsymbol{n}$. The thermal flux is given by

$$
\boldsymbol{J}_{q}^{\text {edge }}=K_{q} \hat{\epsilon} \boldsymbol{n}
$$

with

$$
K_{q}=\frac{s_{B}}{h} \sum_{\alpha} \int_{\varepsilon_{\alpha}}^{\infty}\left(\varepsilon-\mu_{\mathrm{ec}}\right) f\left(\varepsilon, \mu_{\mathrm{ec}}, T_{\mathrm{e}}\right) d \varepsilon,
$$

which is written as

$$
K_{q}=s_{B} \frac{2 \pi l^{2}}{h} \int_{-\infty}^{\mu}\left(\Omega_{0}\left(\mu^{\prime}\right)+T_{\mathrm{e}} s_{0}\left(\mu^{\prime}\right)\right) d \mu^{\prime} .
$$

Derivatives of $K_{n}$ and $K_{q}$ are given by

$$
\begin{aligned}
& \frac{\partial K_{n}}{\partial \mu}=L_{y x}^{11}, \quad \frac{\partial K_{n}}{\partial T_{\mathrm{e}}}=\frac{L_{y x}^{12}}{T_{\mathrm{e}}}, \\
& \frac{\partial K_{q}}{\partial \mu}=K_{y x}^{21}, \quad \frac{\partial K_{q}}{\partial T_{\mathrm{e}}}=\frac{L_{y x}^{22}}{T_{\mathrm{e}}},
\end{aligned}
$$

with

$$
\begin{aligned}
& L_{y x}^{12}=s_{B} \frac{2 \pi l^{2}}{h} T_{\mathrm{e}} s_{0}=K_{y x}^{21}+K_{n}, \\
& L_{y x}^{22}=s_{B} \frac{2 \pi l^{2}}{h} T_{\mathrm{e}}^{2} \int_{-\infty}^{\mu} \frac{\partial s_{0}\left(\mu^{\prime}\right)}{\partial T_{\mathrm{e}}} d \mu^{\prime} .
\end{aligned}
$$

$L_{y x}^{22}$ is also expressed from eqs.(40) and (43) by

$$
L_{y x}^{22}=2 K_{q}+\frac{s_{B}}{h} \sum_{\alpha}\left(\varepsilon_{\alpha}-\mu_{\mathrm{ec}}\right)^{2} f_{\alpha}
$$

\subsection{Measured Fluxes}

\subsubsection{Fluxes through the Sample}

First we consider fluxes through a cross section of the sample along the $x$ direction. Each of the number and thermal fluxes is written as the sum of the bulk and edge contributions.

The number flux, denoted as $J_{n x}^{\mathrm{tr}}$, is given by

$$
J_{n x}^{\operatorname{tr}}=\int_{0}^{\Delta y} j_{n x} d y+J_{n x}^{\operatorname{edge}(\Delta y)}+J_{n x}^{\operatorname{edge}(0)} .
$$

Here $J_{n x}^{\text {edge }(0)}$ and $J_{n x}^{\text {edge }(\Delta y)}$ are the edge current flowing in $y<0$ and that in $y>\Delta y$, respectively. The sum of these edge currents is zero in equilibrium. In nonequilibrium states it is induced by the differences $\Delta \mu=\mu(\Delta y)-\mu(0)$ and $\Delta T_{\mathrm{e}}=T_{\mathrm{e}}(\Delta y)-T_{\mathrm{e}}(0)$, and is written in their first order as

$$
\begin{gathered}
J_{n x}^{\text {edge }(\Delta y)}+J_{n x}^{\text {edge }(0)}=K_{n}(\Delta y)-K_{n}(0) \\
=L_{y x}^{11} \Delta \mu+\frac{L_{y x}^{12}}{T_{\mathrm{e}}} \Delta T_{\mathrm{e}} .
\end{gathered}
$$

The flux density in the bulk region, $j_{n x}$, is given by eq.(31) and is independent of $y$ in the lowest order of $\Delta y$. Therefore $J_{n x}^{\mathrm{tr}}$ is given by

$$
\frac{J_{n x}^{\mathrm{tr}}}{\Delta y}=-L_{x x}^{11} \nabla_{x} \mu_{\mathrm{ec}}+L_{y x}^{11} \nabla_{y} \mu_{\mathrm{ec}}-\frac{L_{x x}^{12}}{T_{\mathrm{e}}} \nabla_{x} T_{\mathrm{e}}+\frac{L_{y x}^{12}}{T_{\mathrm{e}}} \nabla_{y} T_{\mathrm{e}} .
$$

The measured number flux $J_{n x}^{\mathrm{tr}}$ is written only in terms of $\nabla \mu_{\mathrm{ec}}$ and $\nabla T_{\mathrm{e}}$ since it is zero in equilibrium.

The thermal flux $J_{q x}^{\mathrm{tr}}$ is given by

$$
J_{q x}^{\operatorname{tr}}=\int_{0}^{\Delta y} j_{q x} d y+J_{q x}^{\operatorname{edge}(\Delta y) \operatorname{tr}}+J_{q x}^{\text {edge }(0) \operatorname{tr}},
$$

where

$$
J_{q x}^{\text {edge, } \text { tr }}=J_{\epsilon x}^{\text {edge }}-\mu_{\mathrm{ec}}^{0} J_{n x}^{\text {edge }}=J_{q x}^{\text {edge }}+\left(\mu_{\mathrm{ec}}-\mu_{\mathrm{ec}}^{0}\right) J_{n x}^{\text {edge }} .
$$

Here $\mu_{\mathrm{ec}}^{0}$ is a reference electrochemical potential and lies between $\mu_{\mathrm{ec}}(0)$ and $\mu_{\mathrm{ec}}(\Delta y)$. It is the value of the uniform electrochemical potential in a fictitous thin electrode, which is inserted into the sample to measure the fluxes through a cross section at any $x$. In the first order of $\Delta y$, the contribution from edges becomes

$J_{q x}^{\text {edge }(\Delta y) \operatorname{tr}}+J_{q x}^{\text {edge }(0) \operatorname{tr}}=K_{y x}^{21} \Delta \mu+\frac{L_{y x}^{22}}{T_{\mathrm{e}}} \Delta T_{\mathrm{e}}+K_{n} \Delta \mu_{\mathrm{ec}}$,

where $\Delta \mu_{\mathrm{ec}}=\mu_{\mathrm{ec}}(\Delta y)-\mu_{\mathrm{ec}}(0)$. The contribution of the last term, $K_{n} \Delta \mu_{\mathrm{ec}}$, due to the diamagnetic surface current has been first pointed out by Obraztsov. ${ }^{28}$ Then $J_{q x}^{\mathrm{tr}}$ is given by

$\frac{J_{q x}^{\mathrm{tr}}}{\Delta y}=-L_{x x}^{12} \nabla_{x} \mu_{\mathrm{ec}}+L_{y x}^{12} \nabla_{y} \mu_{\mathrm{ec}}-\frac{L_{x x}^{22}}{T_{\mathrm{e}}} \nabla_{x} T_{\mathrm{e}}+\frac{L_{y x}^{22}}{T_{\mathrm{e}}} \nabla_{y} T_{\mathrm{e}}$.

\subsubsection{Fluxes through the Boundary}

Next we consider fluxes along the $y$ direction through each of boundaries at $y=0$ and $y=\Delta y$ into a fictitious electrode outside the sample.

First we use again the coordinates $(\xi, \eta)$ introduced in $\S 4.2$ and write fluxes through a segment of the boundary with length $\Delta \xi$ in terms of the bulk and edge currents. We integrate eq.(4) over an infinitesimal region with width $\Delta \xi$ in $\eta_{\text {edge }}<\eta<\eta_{\text {edge }}+\Delta \eta$. The integration of the left hand side of eq.(4) is of the order of $\Delta \eta$ and is neglected. By considering additionally the number flux through the segment of the boundary, which is denoted as $\Delta J_{n \eta}^{\mathrm{tr}}$, we have

$$
j_{n \eta}\left(\eta_{\text {edge }}\right) \Delta \xi+\left[J_{n \xi}^{\text {edge }}(\xi)-J_{n \xi}^{\text {edge }}(\xi+\Delta \xi)\right]=\Delta J_{n \eta}^{\mathrm{tr}} .
$$

By taking the limit of small $\Delta \xi$, we obtain

$$
\frac{\Delta J_{n \eta}^{\mathrm{tr}}}{\Delta \xi}=j_{n \eta}\left(\eta_{\text {edge }}\right)-\nabla_{\xi} J_{n \xi}^{\text {edge }}
$$

For the thermal flux, we have from eq.(7)

$$
\frac{\Delta J_{q \eta}^{\mathrm{tr}}}{\Delta \xi}=j_{q \eta}\left(\eta_{\text {edge }}\right)-\nabla_{\xi} J_{q \xi}^{\text {edge }}-J_{n \xi}^{\text {edge }} \nabla_{\xi} \mu_{\mathrm{ec}} .
$$

The above formulas are now applied to the boundaries of the sample at $y=0$ and $\Delta y$. At $y=\Delta y$,

$$
\nabla_{x} J_{n x}^{\text {edge }}=\nabla_{x} K_{n}=L_{y x}^{11} \nabla_{x} \mu+\frac{L_{y x}^{12}}{T_{\mathrm{e}}} \nabla_{x} T_{\mathrm{e}},
$$

and the measured number flux $\Delta J_{n y}^{\mathrm{tr}}$ is obtained to be $\frac{\Delta J_{n y}^{\mathrm{tr}}}{\Delta x}=-L_{y x}^{11} \nabla_{x} \mu_{\mathrm{ec}}-L_{x x}^{11} \nabla_{y} \mu_{\mathrm{ec}}-\frac{L_{y x}^{12}}{T_{\mathrm{e}}} \nabla_{x} T_{\mathrm{e}}-\frac{L_{x x}^{12}}{T_{\mathrm{e}}} \nabla_{y} T_{\mathrm{e}}$. 
The same formula is obtained for the boundary at $y=0$. For the thermal flux at $y=\Delta y$,

$$
\nabla_{x} J_{q x}^{\text {edge }}=K_{y x}^{21} \nabla_{x} \mu+\frac{L_{y x}^{22}}{T_{\mathrm{e}}} \nabla_{x} T_{\mathrm{e}},
$$

and the measured thermal flux $\Delta J_{q y}^{\mathrm{tr}}$ is

$$
\frac{\Delta J_{q y}^{\mathrm{tr}}}{\Delta x}=-L_{y x}^{12} \nabla_{x} \mu_{\mathrm{ec}}-L_{x x}^{12} \nabla_{y} \mu_{\mathrm{ec}}-\frac{L_{y x}^{22}}{T_{\mathrm{e}}} \nabla_{x} T_{\mathrm{e}}-\frac{L_{x x}^{22}}{T_{\mathrm{e}}} \nabla_{y} T_{\mathrm{e}},
$$

which is the case both at $y=0$ and at $y=\Delta y$.

\section{Formulation with Transport Fluxes}

\subsection{Transport Flux Densities}

In this section we introduce a transport flux density at each point within the large sample by using the formula for each of the measured fluxes in the narrow system in the previous section. The transport flux densities, denoted as $\boldsymbol{j}_{n}^{\operatorname{tr}}(\boldsymbol{r}, t)$ and $\boldsymbol{j}_{q}^{\operatorname{tr}}(\boldsymbol{r}, t)$, are defined at each point $\boldsymbol{r}$ by

$$
\left(\begin{array}{c}
\boldsymbol{j}_{n}^{\operatorname{tr}}(\boldsymbol{r}, t) \\
\boldsymbol{j}_{q}^{\operatorname{tr}}(\boldsymbol{r}, t)
\end{array}\right)=\left(\begin{array}{cc}
L^{11} & L^{12} \\
L^{21} & L^{22}
\end{array}\right)\left(\begin{array}{c}
-\nabla \mu_{\mathrm{ec}} \\
-T_{\mathrm{e}}^{-1} \nabla T_{\mathrm{e}}
\end{array}\right),
$$

with

$$
\begin{aligned}
L^{11} & =\left(\begin{array}{cc}
L_{x x}^{11} & L_{x y}^{11} \\
L_{y x}^{11} & L_{y y}^{11}
\end{array}\right), L^{12}=\left(\begin{array}{cc}
L_{x x}^{12} & L_{x y}^{12} \\
L_{y x}^{12} & L_{y y}^{12}
\end{array}\right), \\
L^{21} & =\left(\begin{array}{cc}
L_{x x}^{21} & L_{x y}^{21} \\
L_{y x}^{21} & L_{y y}^{21}
\end{array}\right), L^{22}=\left(\begin{array}{cc}
L_{x x}^{22} & L_{x y}^{22} \\
L_{y x}^{22} & L_{y y}^{22}
\end{array}\right),
\end{aligned}
$$

where $L_{y x}^{21}=L_{y x}^{12}$ and, since the system is isotropic in the $x y$ plane, we have the following symmetry relation: $L_{y y}^{i j}=L_{x x}^{i j}$ and $L_{x y}^{i j}=-L_{y x}^{i j}$ with $i=1,2$ and $j=1,2$. The right hand side of eq.(61) is equivalent to that of the formulas of the measured fluxes in the previous section. We note that the coefficients here depend in general on $x, y$, and $t$ through $T_{\mathrm{e}}(\boldsymbol{r}, t)$ and $\mu(\boldsymbol{r}, t)$.

Diagonal and off-diagonal transport coefficients have different microscopic origins: diagonal transport coefficients are due to hopping processes, while off-diagonal ones are due to drift processes. Note also that offdiagonal transport coefficients are written in terms of thermodynamic quantities and do not depend on any transition rates.

It is straightforward to show from eqs. (31), (32), (33), (34), and (61) that the transport flux densities and the total flux densities are related by

$$
\boldsymbol{j}_{n}=\boldsymbol{j}_{n}^{\mathrm{tr}}+\hat{\epsilon} \boldsymbol{\nabla} M_{n}, \quad \boldsymbol{j}_{\epsilon}=\boldsymbol{j}_{\epsilon}^{\mathrm{tr}}+\hat{\epsilon} \boldsymbol{\nabla} M_{\epsilon},
$$

with

$$
M_{n}=-K_{n}, \quad M_{\epsilon}=-\left(K_{q}+\mu_{\mathrm{ec}} K_{n}\right),
$$

where $\boldsymbol{j}_{\epsilon}^{\text {tr }}$ and $\boldsymbol{j}_{q}^{\text {tr }}$ are related by $\boldsymbol{j}_{q}^{\text {tr }}=\boldsymbol{j}_{\epsilon}^{\text {tr }}-\mu_{\mathrm{ec}} \boldsymbol{j}_{n}^{\text {tr }}$, and $K_{n}$ and $K_{q}$ are now to be evaluated at a point $\boldsymbol{r}$ within the sample. The quantity $M_{n}$ in the above equation is proportional to the magnetization $M, M_{n}=-(c / e) M$, from eq.(38). The above equation states that each of the total flux densities, $\boldsymbol{j}_{n}$ and $\boldsymbol{j}_{\epsilon}$, is the sum of the transport flux density and the current density due to spatial variations of the magnetization, which is called the magnetization current density.

\subsection{Equations with Transport Flux Densities}

We now rewrite the equations of conservation, eqs.(4) and (7), in terms of the transport flux densities. Because the divergence of each magnetization current density in eq.(63) is zero, the equations can be written in terms of $\boldsymbol{j}_{n}^{\text {tr }}$ and $\boldsymbol{j}_{q}^{\text {tr }}$ only:

$$
\frac{\partial n}{\partial t}=-\boldsymbol{\nabla} \cdot \boldsymbol{j}_{n}^{\operatorname{tr}},
$$

$$
T_{\mathrm{e}} \frac{\partial s}{\partial t}=-\boldsymbol{\nabla} \cdot \boldsymbol{j}_{q}^{\mathrm{tr}}-\nabla \mu_{\mathrm{ec}} \cdot \boldsymbol{j}_{n}^{\mathrm{tr}}-P_{\mathrm{L}} .
$$

The term $-\nabla \mu_{\mathrm{ec}} \cdot \boldsymbol{j}_{n}^{\mathrm{tr}}$ is of the second order of the current, while the corresponding term in eq.(7), $-\boldsymbol{\nabla} \mu_{\mathrm{ec}} \cdot \boldsymbol{j}_{n}$, contains a first-order contribution due to the drift component in $\boldsymbol{j}_{n}$ when the confining potential is considered.

The equation for the time evolution of the entropy density is obtained from eq.(66) as

$$
\frac{\partial s}{\partial t}=-\nabla \cdot \boldsymbol{j}_{s}+\left(\frac{d s}{d t}\right)_{\mathrm{i}}+\left(\frac{d s}{d t}\right)_{\mathrm{e}},
$$

where

$$
\boldsymbol{j}_{s}=T_{\mathrm{e}}^{-1} \boldsymbol{j}_{q}^{\mathrm{tr}},
$$

is the entropy flux density,

$$
\left(\frac{d s}{d t}\right)_{\mathrm{i}}=-\boldsymbol{j}_{n}^{\mathrm{tr}} \cdot T_{\mathrm{e}}^{-1} \nabla \mu_{\mathrm{ec}}-\boldsymbol{j}_{q}^{\mathrm{tr}} \cdot T_{\mathrm{e}}^{-2} \nabla T_{\mathrm{e}},
$$

is the rate of entropy production per unit area at $\boldsymbol{r}$, and

$$
\left(\frac{d s}{d t}\right)_{\mathrm{e}}=-T_{\mathrm{e}}^{-1} P_{\mathrm{L}}
$$

is due to the heat transfer to the phonon system.

\subsection{Relations between Transport Coefficients}

The Einstein relation tells that the coefficient in front of $\nabla \mu$ and that in front of $\nabla V$ in each of the flux densities are equal. This is satisfied for the transport flux densities since they are written in terms of $\nabla \mu_{\mathrm{ec}}=$ $\nabla \mu+\nabla V$.

The Onsager relation ${ }^{29,30}$ tells that

$$
L_{k l}(B)=L_{l k}(-B)
$$

where $L_{k l}(B)$ is the coefficient in the formula of the $k$ th flux density in front of the $l$ th thermodynamic force. In the present case with the entropy production in eq.(69), the thermodynamic forces ${ }^{1}$ associated with $\boldsymbol{j}_{n}^{\text {tr }}$ and $\boldsymbol{j}_{q}^{\text {tr }}$ are given by

$$
\boldsymbol{F}_{n}=-T_{\mathrm{e}}^{-1} \nabla \mu_{\mathrm{ec}}, \quad \boldsymbol{F}_{q}=-T_{\mathrm{e}}^{-2} \nabla T_{\mathrm{e}},
$$

respectively. Therefore $L_{k l}(B)$ in this case is equal to the corresponding coefficient in eq.(62) except the common factor $T_{\mathrm{e}}^{-1}$. The coefficients in eq.(62) have the following symmetry with respect to the reversal of $\boldsymbol{B}$ :

$$
\begin{gathered}
L_{x x}^{i j}\left(T_{\mathrm{e}}, \mu,-B\right)=L_{x x}^{i j}\left(T_{\mathrm{e}}, \mu, B\right), \\
L_{y x}^{i j}\left(T_{\mathrm{e}}, \mu,-B\right)=-L_{y x}^{i j}\left(T_{\mathrm{e}}, \mu, B\right),
\end{gathered}
$$


with $i=1,2$ and $j=1,2$. Then we confirm that they satisfy the Onsager relation.

In contrast to the coefficients of the transport flux densities, those of the total flux densities in eq.(31), (32), (33), and (34) do not satisfy the Einstein relation nor the Onsager relation. We also note that the coefficient of the drift component of the total thermal flux density, $K_{y x}^{21}$, is proportional to $T_{\mathrm{e}} s_{0}+\Omega_{0}$ instead of $T_{\mathrm{e}} s_{0}$ [eq.(28)], while the corresponding coefficient of the transport flux density, $L_{y x}^{12}$, is proportional to $T_{\mathrm{e}} s_{0}$ [eq.(44)] and this transport thermal flux density divided by $T_{\mathrm{e}}$ accords with the definition of the entropy flux density.

Smrčka and Streda ${ }^{31}$ have proved that the transport coefficients for number and energy fluxes in quantizing magnetic fields are given by integrals of the zerotemperature conductivity tensor in the case of elastic scatterings (the relation for number and thermal fluxes has been written in ref.32). The same relation holds in the present case. Each coefficient $L_{\alpha \beta}^{i j}$ with $i=1,2$, $j=1,2, \alpha=x, y$, and $\beta=x, y$ can be expressed by

$$
L_{\alpha \beta}^{i j}=\int_{-\infty}^{\infty} d \varepsilon\left(-\frac{\partial f}{\partial \varepsilon}\right)\left(\varepsilon-\mu_{\mathrm{ec}}\right)^{i+j-2} L_{\alpha \beta}^{11(0)}(\varepsilon),
$$

in terms of the zero-temperature coefficients

$$
\begin{gathered}
L_{x x}^{11(0)}(\varepsilon)=L_{y y}^{11(0)}(\varepsilon)=\sum_{\alpha} D_{\alpha} \delta\left(\varepsilon-\varepsilon_{\alpha}\right), \\
L_{y x}^{11(0)}(\varepsilon)=-L_{x y}^{11(0)}(\varepsilon)=\left(s_{B} / h\right) \sum_{\alpha} \theta\left(\varepsilon-\varepsilon_{\alpha}\right),
\end{gathered}
$$

where $\theta(\varepsilon)=0$ for $\varepsilon<0$ and $\theta(\varepsilon)=1$ for $\varepsilon>0$.

\section{Linear-Response Ettingshausen Effect}

As an illustration, we apply the present theory with the transport fluxes to the Ettingshausen effect, in which the gradient of the electron temperature is developed in the direction perpendicular to the current in the presence of the magnetic field. A spatial variation of the electron temperature in the Ettingshausen effect in a quantum Hall system has been studied previously in the nonlinear transport regime. ${ }^{18}$ Here we make a linear-response calculation and investigate spatial variations and quantum oscillations of the electron temperature.

\subsection{Model and Equations}

We consider a two-dimensional system in the region $-W / 2<y<W / 2$. We employ the zero edge-width model in $\S 4.1$. The following two cases are studied. In the first case the width of the sample $W$ is comparable to or larger than the length scale of the electron-temperature variation $\lambda$. The formula of $\lambda$ is given below. In the second case $W$ is much smaller than $\lambda$, and here only terms in the lowest order of $W / \lambda$ are retained.

We use eqs.(65) and (66) and restrict the calculation to the linear-response regime and to steady states. We also assume a uniformity along $x$ where flux densities and thermodynamic quantities have no dependence on $x$. The exception is $\mu_{\mathrm{ec}}$ which has a constant gradient along $x$. The gradient $\nabla_{x} \mu_{\mathrm{ec}}$ is also independent of $y$ since $\nabla_{y} \nabla_{x} \mu_{\mathrm{ec}}=\nabla_{x} \nabla_{y} \mu_{\mathrm{ec}}=0$, and is equal to $\nabla_{x} V=$ $e E_{x}$. Then the equations become

$$
\nabla_{y} j_{n y}^{\mathrm{tr}}=0
$$

$$
\nabla_{y} j_{q y}^{\mathrm{tr}}+P_{\mathrm{L}}=0 .
$$

We employ the simplest model of the energy $\operatorname{loss} P_{\mathrm{L}}$ :

$$
P_{\mathrm{L}}=C_{\mathrm{p}}\left[T_{\mathrm{e}}(y)-T_{\mathrm{L}}\right],
$$

where $C_{\mathrm{p}}$ is a constant. The boundary conditions at $y=$ $\pm W / 2$ are

$$
j_{n y}^{\mathrm{tr}}=0, \quad j_{q y}^{\mathrm{tr}}=0,
$$

since the fluxes to the outside of the sample are absent $\left(\Delta J_{n y}^{\mathrm{tr}}=0\right.$ and $\left.\Delta J_{q y}^{\mathrm{tr}}=0\right)$ in eqs.(58) and (60). Using eq.(78) and (81) we have

$$
j_{n y}^{\operatorname{tr}}=0, \quad-W / 2<y<W / 2 .
$$

\subsection{Spatial Variations}

We substitute $\nabla_{x} \mu_{\mathrm{ec}}=e E_{x}$ and $\nabla_{x} T_{\mathrm{e}}=0$ into the formula of $j_{q y}^{\text {tr }}$, and use eq.(82) to eliminate $\nabla_{y} \mu_{\mathrm{ec}}$. Then we obtain

$$
j_{q y}^{\mathrm{tr}}=-A_{y x}^{21} e E_{x}-A_{y y}^{22} \nabla_{y} T_{\mathrm{e}}
$$

with

$$
\begin{gathered}
A_{y x}^{21}=L_{y x}^{21}-L_{y y}^{21}\left(L_{y y}^{11}\right)^{-1} L_{y x}^{11}, \\
A_{y y}^{22}=\left[L_{y y}^{22}-L_{y y}^{21}\left(L_{y y}^{11}\right)^{-1} L_{y y}^{12}\right] T_{\mathrm{L}}^{-1} .
\end{gathered}
$$

The transport coefficients are constant in the bulk region in the linear-response regime. By substituting eq.(83) into eq.(79), we obtain the equation for $T_{\mathrm{e}}(y)$ :

$$
A_{y y}^{22} \nabla_{y}^{2} T_{\mathrm{e}}=C_{\mathrm{p}}\left(T_{\mathrm{e}}-T_{\mathrm{L}}\right)
$$

with the boundary condition:

$$
j_{q y}^{\mathrm{tr}}=-A_{y x}^{21} e E_{x}-A_{y y}^{22} \nabla_{y} T_{\mathrm{e}}=0, y= \pm W / 2 .
$$

This boundary condition immediately shows that the electric field $E_{x}$ along the current induces the temperature gradient $\nabla_{y} T_{\mathrm{e}}$. Solving the equation, we find the spatial variation of $T_{\mathrm{e}}$ to be

$$
T_{\mathrm{e}}(y)-T_{\mathrm{L}}=T_{0}\left[e^{-(y+W / 2) / \lambda}-e^{(y-W / 2) / \lambda}\right] .
$$

The relaxation length of the $T_{\mathrm{e}}$ deviation is found to be

$$
\lambda=\left(A_{y y}^{22} / C_{\mathrm{p}}\right)^{1 / 2},
$$

where $A_{y y}^{22}>0$ as is proved from the Schwarz inequality. The magnitude of the $T_{\mathrm{e}}$ deviation is

$$
T_{0}=\left(\lambda A_{y x}^{21} / A_{y y}^{22}\right)\left(1+e^{-W / \lambda}\right)^{-1} e E_{x} .
$$

If we assume that $D_{\alpha}=(2 N+1) D_{0}, T_{0} / E_{x}$ depends on $D_{0}$ and also on $C_{\mathrm{p}}$.

\subsection{Quantum Oscillations}

In order to obtain a universal result independent of $D_{0}$ and $C_{\mathrm{p}}$, we consider $\nabla_{y} T_{\mathrm{e}} / \nabla_{y} \mu_{\mathrm{ec}}$ in the limit of $W \ll \lambda$. In this limit the $y$ dependence of $\nabla_{y} T_{\mathrm{e}}$ and $\nabla_{y} \mu_{\mathrm{ec}}$ are negligible from eqs.(88) and (82), respectively. We use $\nabla_{x} T_{\mathrm{e}}=0$ again and eliminate $\nabla_{x} \mu_{\mathrm{ec}}$ by using $j_{n y}^{\mathrm{tr}}=0$. We then obtain

$$
j_{q y}^{\mathrm{tr}}=-B_{y y}^{21} \nabla_{y} \mu_{\mathrm{ec}}-B_{y y}^{22} \nabla_{y} T_{\mathrm{e}} .
$$

with

$$
\begin{gathered}
B_{y y}^{21}=L_{y y}^{21}-L_{y x}^{21}\left(L_{y x}^{11}\right)^{-1} L_{y y}^{11}, \\
B_{y y}^{22}=\left[L_{y y}^{22}-L_{y x}^{21}\left(L_{y x}^{11}\right)^{-1} L_{y y}^{12}\right] T_{\mathrm{L}}^{-1} .
\end{gathered}
$$




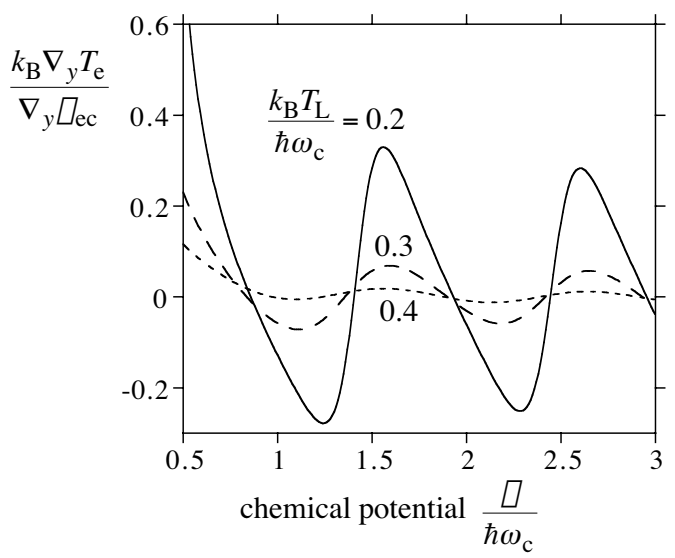

Fig. 1. Electron temperature gradient $\nabla_{y} T_{\mathrm{e}}$ perpendicular to the current divided by the Hall field $\nabla_{y} \mu_{\mathrm{ec}} / e$ ( $\mu_{\mathrm{ec}}$ : the electrochemical potential) is plotted as a function of the chemical potential $\mu$ for several values of the lattice temperature $T_{\mathrm{L}}$.

From the boundary condition $j_{q y}^{\text {tr }}=0$, we find

$$
\nabla_{y} T_{\mathrm{e}} / \nabla_{y} \mu_{\mathrm{ec}}=-B_{y y}^{21} / B_{y y}^{22} .
$$

Here we assume that $D_{\alpha}=(2 N+1) D_{0}$. Then we find that $\nabla_{y} T_{\mathrm{e}} / \nabla_{y} \mu_{\mathrm{ec}}$ is independent of $D_{0}$ and $C_{\mathrm{p}}$. The dimensionless quantity $k_{\mathrm{B}} \nabla_{y} T_{\mathrm{e}} / \nabla_{y} \mu_{\mathrm{ec}}$ is a universal function of $\mu / \hbar \omega_{\mathrm{c}}$ and $k_{\mathrm{B}} T_{\mathrm{L}} / \hbar \omega_{\mathrm{c}}$, which is plotted in Fig.1. Here the spin splitting is neglected. We find that the sign of $\nabla_{y} T_{\mathrm{e}}$ exhibits quantum oscillations as a function of $\mu$ at $k_{\mathrm{B}} T_{\mathrm{L}}<\hbar \omega_{\mathrm{c}}$. Detailed analysis of the Ettingshausen effect will be given elsewhere.

\section{Discussion}

\subsection{Thermoelectric-Transport Theory}

Nearly half a century ago, the thermoelectric coefficients in a magnetic field have been calculated quantummechanically (the references are cited in some papers $^{28,34,35}$ ). Several authors encountered a difficulty of violating the Einstein relation and the Onsager relation when calculating the coefficients for the local current density. This problem was due to the confusion of the local current density with the averaged current density, which is defined as the measured current through a sample divided by a cross section of the sample. The Einstein relation and the Onsager relation should be applied to the measured current, or to its averaged density, and the local current density is different from the averaged current density in a magnetic field due to the presence of the diamagnetic surface current (a description is given in a textbook ${ }^{27}$ and a review paper $\left.{ }^{35}\right)$. Obraztsov ${ }^{28,34}$ has shown explicitly that the Onsager relation holds for the averaged current density by calculating the total current through the sample including the diamagnetic surface current in the case of three-dimensional electron systems. He has also shown ${ }^{34}$ that the thermoelectric coefficients in the absence of disorder can be expressed in terms of the entropy.
A current density, which satisfies both the Einstein relation and the Onsager relation, can be defined also at each point in a sample. It has been defined by $\boldsymbol{j}_{\text {cond }}=$ $\boldsymbol{j}-c \boldsymbol{\nabla} \times \boldsymbol{M}$ and has been called the conduction current density, ${ }^{27,35}$ the macroscopic current density, ${ }^{28}$ or the transport current density. ${ }^{25}$ Here $\boldsymbol{j}$ is the local current density and $c \boldsymbol{\nabla} \times \boldsymbol{M}$ is the magnetization current density with the magnetization $\boldsymbol{M}$. The current density $\boldsymbol{j}_{\text {cond }}$ is zero in equilibrium by definition.

Later the discovery of the quantum Hall effect ${ }^{3,4}$ has stimulated theoretical ${ }^{25,33,36-44}$ and experimental ${ }^{45,46}$ studies on thermoelectric effects in two-dimensional systems in strong magnetic fields. The edge current in a two-dimensional system corresponds to the surface current in a three-dimensional system. The thermoelectric power has been calculated in a two-dimensional system of noninteracting electrons in a quantizing magnetic field in the absence of disorder ${ }^{36,37}$ and in the presence of disorder ${ }^{38,42}$ for short-range scatterings in the selfconsistent Born approximation. ${ }^{26}$ Effects of the localization of wave function on the thermoelectric transport have been studied ${ }^{33}$ within the classical-drift model. ${ }^{20-22}$ Effects of the interaction between electrons on the thermoelectric transport have also been studied. ${ }^{25}$

In this paper we have defined a number transport flux density and an energy transport flux density from the corresponding measured fluxes in a narrow wire and shown that each transport flux density is equal to the total flux density minus the magnetization current density, namely that corresponding to $\boldsymbol{j}-c \boldsymbol{\nabla} \times \boldsymbol{M}$. Note that the current in this paper is due to only the orbital-center motion as in refs. $33,34,37$, while those in refs. $25,27,28,35$ are due to both the orbital-center and relative motions. The localization has been taken into account within the classical-drift model, ${ }^{20-22}$ and the transport coefficients obtained in the present paper eq.(62) reduces to those obtained by Grunwald and Hajdu ${ }^{33}$ in the absence of hopping processes. The transport coefficients in the present paper are generalized so that they are applicable to a nonlinear transport regime within the local equilibrium by including their dependences on the local electron temperature and the local chemical potential.

\subsection{Hot-Electron Theory and Thermohydrodynamics}

One of the most typical nonlinear effects is the electron heating. Uchimura and Uemura ${ }^{47}$ have applied the hot-electron theory and the self-consistent Born approximation ${ }^{26}$ to explain the electric-field dependence of the diagonal conductivity, observed by Kawaji and Wakabayashi, ${ }^{48}$ in two-dimensional systems in quantizing magnetic fields.

Shortly after the discovery of the quantum Hall effect, the breakdown of the quantum Hall effect has been found, ${ }^{8-10}$ in which $\rho_{x x}$ increases by several orders of magnitude when the current is increased up to a critical value. As a mechanism of the breakdown, a hot-electron model, ${ }^{8,49,50}$ has been proposed, which combines the electron heating and the strong $T_{\mathrm{e}}$ dependence of $\rho_{x x}$. Later the hot-electron model has been supported by the observed spatial evolutions of $\rho_{x x} \cdot{ }^{11-13}$

Gurevich and Mints ${ }^{49}$ have proposed a hydrodynamic 
equation based on the hot-electron model to calculate spatio-temporal variations of $T_{\mathrm{e}}$ in quantum Hall systems. Their equation, however, is restricted to onedimensional variations of $T_{\mathrm{e}}$, and the thermal flux due to the drift motion is missing.

The previous theory by one of the present authors ${ }^{18}$ has proposed a set of hydrodynamic equations for twodimensional variations of $T_{\mathrm{e}}$ and $\mu_{\mathrm{ec}}$ as well as a model of flux densities due to drift and hopping processes, which is a generalization of the theory for one-dimensional variations by the same author. ${ }^{51}$ In this theory ${ }^{18}$ however, the drift thermal flux density was not correct: the drift thermal flux density in eq.(3.13) there was $\boldsymbol{j}_{q}^{\text {drift }}-\boldsymbol{j}_{q 0}^{\text {drift }}$ where $\boldsymbol{j}_{q 0}^{\text {drift }}$ is the drift thermal flux density at $T_{\mathrm{e}}=0$, but it should be $\boldsymbol{j}_{q}^{\mathrm{drift}}$. Equation (3.13) in ref.18 should be replaced by eq.(7) in the present paper and boundary conditions eq.(3.14) should be corrected to $\boldsymbol{j}_{n}^{\text {tr }} \cdot \boldsymbol{n}_{\mathrm{b}}=0$, $\boldsymbol{j}_{q}^{\mathrm{tr}} \cdot \boldsymbol{n}_{\mathrm{b}}=0$ where $\boldsymbol{n}_{\mathrm{b}}$ is the unit vector perpendicular to the boundary.

\section{Conclusions}

We have developed a theory of thermohydrodynamics in quantum Hall systems to study spatio-temporal variations in the linear- and nonlinear-transport regime in the local-equilibrium approximation. A nonlinear effect has been taken into account through dependences of the transport coefficients on the local electron temperature and the local chemical potential. This theory can be used to investigate, for example, the electron temperature distribution in the vicinity of the breakdown of the quantum Hall effect in a system with compressible and incompressible strips.

Quantum Hall systems possess several unique features. The formation of the Landau levels gives rise to quantum oscillations of thermoelectric properties as a function of the filling factor. The activation energy for the transport plays an essential role in producing the bistability in the breakdown of the quantum Hall effect. The large drift current is responsible for the observed long relaxation length of the electron temperature. ${ }^{11-13}$ These features will continue to provide a variety of unique thermohydrodynamic phenomena in quantum Hall systems.

\section{Acknowledgments}

The authors would like to thank T. Ise, K. Shimoyama, T. Maeda, T. Nakagawa, and S. Kanamaru for valuable discussions. This work was supported in part by the Grant-in-Aid for Scientific Research (C) from Japan Society for the Promotion of Science.

1) S.R. de Groot and P. Mazur: Non-equilibrium Thermodynamics (North-Holland, Amsterdam, 1962).

2) E. Schöll: Nonlinear Spatio-Temporal Dynamics and Chaos in Semiconductors (Cambridge University Press, Cambridge, 2001).

3) K. von Klitzing, G. Dorda and M. Pepper: Phys. Rev. Lett. 45 (1980) 494.

4) S. Kawaji and J. Wakabayashi: in Physics in High Magnetic Fields (Springer, Berlin, 1981) p. 284.

5) D. B. Chklovskii, B. I. Shklovskii, and L. I. Glazman: Phys. Rev. B 46 (1992) 4026.
6) E. Ahlswede, P. Weitz, J. Weis, K. von Klitzing, and K. Eberl: Physica B 298 (2001) 562.

7) K. Güven and R. R. Gerhardts: Phys. Rev. B 67 (2003) 115327.

8) G. Ebert, K. von Klitzing, K. Ploog and G. Weimann: J. Phys. C 16 (1983) 5441.

9) M. E. Cage, R. F. Dziuba, B. F. Field, E. R. Williams, S. M. Girvin, A. C. Gossard, D. C. Tsui and R. J. Wagner: Phys. Rev. Lett. 51 (1983) 1374.

10) F. Kuchar, G. Bauer, G. Weimann and H. Burkhard: Surf. Sci. 142 (1984) 196.

11) S. Komiyama, Y. Kawaguchi, T. Osada and Y. Shiraki: Phys. Rev. Lett. 77 (1996) 558.

12) I. I. Kaya, G. Nachtwei, K. von Klitzing and K. Eberl: Phys. Rev. B 58 (1998) R7536.

13) I. I. Kaya, G. Nachtwei, K. von Klitzing and K. Eberl: Europhys. Lett. 46 (1999) 62.

14) F. J. Ahlers, G. Hein, H. Scherer, L. Bliek, H. Nickel, R. Lösch and W. Schlapp: Semicond. Sci. Technol. 8 (1993) 2062.

15) B.E. Sagol, G. Nachtwei, I. I. Kaya, K. von Klitzing and K. Eberl: Proc. 25th Int. Conf. Phys. Semicond., eds. N. Miura and T. Ando (Springer, Berlin, 2001) p. 959.

16) Y. Kawano and S. Komiyama: Phys. Rev. B 61 (2000) 2931.

17) Y. Kawano and S. Komiyama: Phys. Rev. B 68 (2003) 085328.

18) H. Akera: J. Phys. Soc. Jpn. 71 (2002) 228.

19) J. A. Nixon and J. H. Davies: Phys. Rev. B 41 (1990) 7929.

20) S. V. Iordansky: Solid State Commun. 43 (1982) 1.

21) R. E. Prange and R. Joynt: Phys. Rev. B 25 (1982) 2943.

22) S. M. Apenko and Yu. E. Lozovik: J. Phys. C 18 (1985) 1197.

23) H. Akera: J. Phys. Soc. Jpn. 69 (2000) 3174.

24) T. Ise and H. Akera: J. Phys. Soc. Jpn. 71 (2002) 1712.

25) N. R. Cooper, B. I. Halperin and I. M. Ruzin: Phys. Rev. B 55 (1997) 2344.

26) T. Ando and Y. Uemura: J. Phys. Soc. Jpn. 36 (1974) 959.

27) L. D. Landau and E. M. Lifshitz: Electrodynamics of Continuous Media (Pergamon Press, Oxford, 1960) §29.

28) Yu. N. Obraztsov: Fiz. Tverd. Tela (Leningrad) 6 (1964) 414 [Sov. Phys. Solid State 6 (1964) 331].

29) L. Onsager: Phys. Rev. 37 (1931) 405.

30) L. Onsager: Phys. Rev. 38 (1931) 2265.

31) L. Smrčka and P. Středa: J. Phys. C 10 (1977) 2153.

32) P. Středa and H. Oji: Phys. Lett. 102A (1984) 201.

33) A. Grunwald and J. Hajdu: Solid State Commun. 63 (1987) 289.

34) Yu. N. Obraztsov: Fiz. Tverd. Tela (Leningrad) 7 (1965) 573 [Sov. Phys. Solid State 7 (1965) 455].

35) C. Herring: J. Phys. Soc. Jpn. 21, Suppl. (1966) v.

36) S. P. Zelenin, A. S. Kondrat'ev, and A. E. Kuchma: Fiz. Tekh. Poluprovodn. 16 (1982) 551 [Sov. Phys. Semicond. 16 (1982) 355].

37) S. M. Girvin and M. Jonson: J. Phys. C 15 (1982) L1147.

38) M. Jonson and S. M. Girvin: Phys. Rev. B 29 (1984) 1939.

39) P. Středa: J. Phys. C 16 (1983) L369.

40) P. Středa: Phys. Status Solidi. (b) 125 (1984) 849.

41) H. Oji: Phys. Rev. B 29 (1984) 3148.

42) H. Oji: J. Phys. C 17 (1984) 3059.

43) H. Oji and P. Středa: Phys. Rev. B 31 (1985) 7291.

44) W. Zawadzki and R. Lassnig: Surf. Sci. 142 (1984) 225.

45) H. Obloh, K. von Klitzing, and K. Ploog: Surf. Sci. 142 (1984) 236.

46) B. L. Gallagher and P. N. Butcher: in Handbook on Semiconductors, edited by P. T. Landsberg (North-Holland, Amsterdam, 1992), Vol. 1, p. 721.

47) N. Uchimura and Y. Uemura: J. Phys. Soc. Jpn. 47 (1979) 1417.

48) S. Kawaji and J. Wakabayashi: Surf. Sci. 58 (1976) 238.

49) A. Vl. Gurevich and R. G. Mints: Pis'ma Zh. Eksp. Teor. Fiz. 39 (1984) 318 [JETP Lett. 39 (1984) 381].

50) S. Komiyama, T. Takamasu, S. Hiyamizu and S. Sasa: Solid State Commun. 54 (1985) 479.

51) H. Akera: J. Phys. Soc. Jpn. 70 (2001) 1468. 